\title{
STATISTICAL CRITERIA TO ESTABLISH BIOASSAY PROGRAMS
}

\author{
Jesus Lopez-Fidalgo* and Guillermo Sanchez ${ }^{\dagger}$
}

\begin{abstract}
A statistical study of fitting log-normal distributions to air sampling concentrations and random intakes is reported in this paper. An improved method on the approximation of a sum of log-normal distributions to a log-normal distribution is used to forecast bioassay results and their uncertainties. These methods can be used in the design and implementation of air control monitoring and bioassay programs for introducing statistical criteria to determine when a bioassay is required as well as the frequency.
\end{abstract}

Health Phys. 89(4):333-338; 2005

Key words: biokinetics; modeling, biological factors; air sampling; intake, radionuclide

\section{INTRODUCTION}

AIR SAMPLERS frequently are used to track exposure and to calculate intakes for workers exposed to radioactive aerosols, e.g., in uranium and plutonium processing plants. A bioassay program also is applied periodically to the workers monitored using lung counters and urine samples, and less frequently with fecal samples. The biokinetic model can be applied to establish a relationship between the individual intake and the bioassay program frequency. In fact, using the models and criteria in reports by the International Commission on Radiological Protection (ICRP), lung retention and urine and fecal excretion can be evaluated using the differential equation

$$
\mathbf{q}^{\prime}(t)=\mathbf{A} \mathbf{q}(t)+\mathbf{b}(t),
$$

where $\mathbf{A}=\mathbf{K}-\lambda \mathbf{I}$, and $\mathbf{K}$ is the matrix of transfer rates with elements $k_{\mathrm{ij}}$ representing the constant fractional transfer rate from compartment $j$ to $i(i \neq j)$ and $k_{\mathrm{ii}}$ representing the negative of the total removal rate constants, that is the

* Department of Statistics, University of Salamanca, Plaza de los Caidos s/n, 37008-Salamanca, Spain; ${ }^{\dagger}$ ENUSA Industrias Avanzadas S.A. Fabrica de Juzbado, Apdo 328, 37080-Salamanca, Spain, and Department of Foundations of Economical Analysis, University of Salamanca, Spain.

For correspondence or reprints contact: Jesus Lopez-Fidalgo, Faculdad de Ciencias, Universidad de Salamanca, Plaza de los Caidos s/n, 37008-Salamanca, Spain, or email at fidalgo@usal.es.

(Manuscript received 8 January 2004; revised manuscript 15 March 2005, accepted 16 May 2005)

0017-9078/05/0

Copyright (C) 2005 Health Physics Society rate constants over $j$ plus the radiological rate constant, $\lambda$. The vector $\mathbf{q}(t)=\left[q_{\mathrm{l}}(t), \ldots, q_{\mathrm{i}}(t), \ldots, q_{\mathrm{n}}(t)\right]^{T}$ represents the function of content in each compartment $i$ of an $n$-compartmental system at time $t$ and $\mathbf{b}(t)=\left[b_{1}(t), \ldots\right.$, $\left.b_{\mathrm{i}}(t), \ldots, b_{\mathrm{n}}(t)\right]^{T}$ represents the input function in each compartment $i$ of the system at time $t$.

The solution of eqn (1) is given by eqn (2), where $\mathbf{q}_{0}=\left[q_{1}(0), \ldots, q_{\mathrm{i}}(0), \ldots, q_{\mathrm{n}}(0)\right]^{T}$ is the content in each compartment $i$ at time $t=0$,

$$
\mathbf{q}(t)=e^{\mathbf{A} t} \mathbf{q}_{0}+\int_{0}^{t} e^{\mathbf{A}(t-\tau)} \mathbf{b}(\tau) d \tau .
$$

For a single (or acute) intake eqn (2) becomes

$$
\mathbf{q}(t)=e^{\mathbf{A} t} \mathbf{q}_{0} .
$$

When the matrix $\mathbf{A}$ is diagonalizable (all current ICRP models, compiled in ICRP Database of Dose Coefficients 2001, have this characteristic), eqn (3) gives $q_{\mathrm{i}}(t)$ as a sum of exponentials $q_{\mathrm{i}}(t)=q_{\mathrm{r}}(0) \sum_{\mathrm{v}=1}^{\mathrm{l}_{\mathrm{i}}} \mathrm{c}_{\mathrm{v}}^{(\mathrm{i})} \exp \left(-d_{\mathrm{v}}^{(\mathrm{i})} t\right)$. Thus, the lung and whole body retention for a single intake $\Sigma_{\mathrm{i}} q_{\mathrm{i}}(0)=$ 1 , as well as the daily urine and fecal excretion, after a time $t$, are sums of contents of several compartments. Therefore, they follow the same pattern,

$$
r(t)=\sum_{v=1}^{l} c_{v} \exp \left(-d_{v} t\right),
$$

where $r(t)$ is usually called the intake retention function (e.g., Skrable et al. 1988) and the value of this function at a specific time $t$ is known as the Intake Retention Fraction (IRF) (e.g., Potter 2002). If the intake is continuously constant the intake retention function has a similar form of eqn (4). IRF values for single intake are tabulated for many radioisotopes, e.g., ICRP 78 (1997) and Potter (2002). Analytic expressions of $r(t)$ and IRF values for acute and chronic intakes can be obtained using BIOKMOD (Sanchez 2005; Sanchez and Lopez-Fidalgo 2003). IRF values are often used in bioassay programs.

\footnotetext{
BIOKMOD is available at http://www3.enusa.es/webMathematica/ Public/biokmod.html.
} 
The individual daily intake for an occupational worker is usually a random variable (r.v.). This means that $\mathbf{b}(t)$ in eqn (1) is a r.v. The solution of eqn (1) in situations where $\mathbf{b}(t)$ is a r.v. has been studied, e.g., by Kercher and Robison (1993). We are interested in the case when a random intake can be fitted to a known probability distribution function (pdf) to provide probability bands around the function $r(t)$ for planning the bioassay program.

\section{METHODS}

Let us consider a worker exposed to an environment of airborne radioactivity. The worker inhales a quantity $I_{\mathrm{j}}$ each day $j$. Although the intake happens during a few hours every day, from a practical point of view it can be assumed that $I_{\mathrm{j}}$ is an acute intake. This is a usual assumption very convenient for our purpose. After a time $t$ the worker will have been exposed to multiple acute intakes $I_{1}, \ldots, I_{\mathrm{t}}$. Then the lung and the whole body retention as well as the daily urine and fecal excretion after a time $t$ will follow the pattern of eqn (5) where the time is considered as a discrete variable measured in days and $s_{\mathrm{j}}(t)=r(t-j+1)$ :

$$
\begin{aligned}
y(t)=I_{1} r(t)+I_{2} r & (t-1)+\cdots+I_{\mathrm{t}} r(1) \\
& =\sum_{j=1}^{l} I_{j} r(t-j+1)=\sum_{j=1}^{l} I_{j} s_{j}(t) .
\end{aligned}
$$

We are interested in studying $y(t)$ and its uncertainties when $I_{\mathrm{j}}$ is a r.v. as described here. If the pdf of this r.v. can be fitted to the pdf of a known distribution, then some theoretical characteristics of the pdf may be applicable. With this purpose we have analyzed the intake data of a group of workers from Juzbado Fuel Fabrication Plant who were exposed over a long period of time (usually a few years) to chronic intake. The Juzbado factory makes uranium fuel assemblies for light water reactors. This process requires manipulating the powder of enriched uranium oxides $\left(<5 \%\right.$ of $\left.{ }^{235} \mathrm{U}\right)$. The "Ceramic Zone" is the workshop where airborne radioactive particles may be released into the environment and there is a potential radiological hazard of internal contamination. This zone is divided into a few areas (for pressing, sintering, grinding, etc.). The exposure conditions inside each area are considered to be similar. We have found that the daily intake $I_{\mathrm{j}}$ for workers performing their activities in the same area for a long period of time (greater than 400 working days) can be modeled using a log-normal distribution $L N\left(\mu, \sigma^{2}\right)$, where $\mu$ and $\sigma^{2}$ are the mean and variance of the corresponding normal distribution. The pdf of the log-normal distribution is given by eqn (6):

$$
f(x)=\left\{\begin{array}{cl}
\frac{1}{\sigma \mathrm{x} \sqrt{2 \pi}} \exp \left[-\frac{1}{2}\left(\frac{\ln x-\mu}{\sigma}\right)^{2}\right] & \text { if } x>0 \\
0 & \text { if } x \leq 0 .
\end{array}\right.
$$

The mean and variance of the log-normal distribution are

$$
\mu_{X}=e^{\mu+\sigma^{2} / 2}, \quad \sigma_{X}^{2}=e^{2 \mu+\sigma^{2}}\left(e^{\sigma^{2}}-1\right),
$$

while the mean and variance of the associated normal distribution $(\ln X)$ are

$$
\mu=\ln \left\{\mu_{X}\left[1+\left(\frac{\sigma_{X}}{\mu_{X}}\right)^{2}\right]^{-1 / 2}\right\}, \quad \sigma^{2}=\ln \left[1+\left(\frac{\sigma_{X}}{\mu_{X}}\right)^{2}\right] .
$$

The following estimators derived from the transformation to the normal distribution are widely used in practice:

$$
\mu_{e}=\frac{1}{N} \sum_{i} \ln x_{i}, \quad \sigma_{e}^{2}=\frac{1}{N} \sum_{i}\left(\ln x_{i}-\mu_{e}\right)^{2},
$$

where $N$ is the total number of observations $x_{\mathrm{i}}$ from the log-normal distribution $(i=1, \ldots, N)$.

In our case the daily intake for a specific worker $I_{\mathrm{j}}$ plays the role of $x$ and it has to be taken into account that for non-working days, Saturdays, and Sundays, $I_{\mathrm{j}}$ is assumed to be zero. Thus, in eqn (5) for each $j$ corresponding to a working day, $s_{\mathrm{j}}(t) I_{\mathrm{j}}$ is the product of a constant and a r.v. It is well known that a r.v. proportional to a log-normal distribution also is lognormal. Therefore, $s_{\mathrm{j}}(t) I_{\mathrm{j}}$ for working days follows a log-normal distribution. The sum of log-normal variables is not a known distribution, but there are some approximations to log-normal distributions (Fenton 1960; Schwartz and Yeh 1982). One of the simplest is Fenton-Wilkinson's approximation (Abu-Dayya and Beaulieu 1994; Ho 1995). This approximation has been proven accurate under certain conditions (e.g., Cardieri and Rappaport 2001) as is the case here for independent r.v., means not significantly different, and similar variances. In particular, Fenton-Wilkinson's approximation for $y(t)$ in eqn (5) provides a lognormal distribution $L N\left[\mu(t), \sigma^{2}(t)\right]$ with first and second moments about the origin given by

$$
u_{1}(t)=E[y(t)]=\sum_{j} \exp \left[m_{j}(t)+\mu+\sigma^{2} / 2\right]
$$




$$
\begin{aligned}
u_{2}(t)=E\left[y(t)^{2}\right]=\sum_{j} \exp \left\{2\left[m_{j}(t)+\mu+\sigma^{2}\right]\right\} \\
+\sum_{j \neq j^{\prime}} \sum_{j^{\prime}} \exp \left[m_{j}(t)+m_{j}(t)+2 \mu+\sigma^{2}\right],
\end{aligned}
$$

where $m_{\mathrm{j}}(t)=\ln [r(t-j+1)]$. Substituting $m_{\mathrm{j}}(t)$ in eqn (10) and eqn (11) we obtain

$$
\begin{gathered}
u_{1}(t)=e^{\mu+\sigma^{2} / 2} \sum_{j} r(j)=\mu_{I} \sum_{j} r(j), \\
u_{2}(t)=u_{1}(t)^{2}+e^{2 \mu+\sigma^{2}}\left(e^{\sigma^{2}}-1\right) \sum_{j} r^{2}(j) \\
=u_{1}(t)^{2}+\sigma_{I}^{2} \sum_{j} r^{2}(j),
\end{gathered}
$$

where $\mu_{\mathrm{I}}$ and $\sigma_{\mathrm{I}}^{2}$ are the mean and variance of the log-normal distribution of the intake obtained using eqn (7). Thus, the approximated log-normal distribution for eqn (5) has the following parameters:

$$
\begin{aligned}
\mu(t)=2 \ln u_{1}(t)-\frac{1}{2} \ln u_{2}(t) & \\
\sigma^{2}(t) & =\ln u_{2}(t)-2 \ln u_{1}(t) .
\end{aligned}
$$

An approximation of the cumulative distribution function (cdf) of $y(t)$ is then

$$
P\left[y(t) \leq y_{0}\right] \approx \Phi\left[\frac{\ln y_{0}-\mu(t)}{\sigma(t)}\right],
$$

where $\Phi$ is the normal cdf. Probability bands for $y(t)$ can be constructed for a fixed probability $\gamma$ :

$$
P\left[y(t) \leq u_{U}(t)\right]=\frac{1+\gamma}{2}, \quad P\left[y(t) \leq u_{L}(t)\right]=\frac{1-\gamma}{2},
$$

that is, $u_{\mathrm{L}}(t) \approx \exp [\mu(t)-z \sigma(t)]$ and $u_{\mathrm{U}}(t) \approx$ $\exp [\mu(t)+z \sigma(t)]$, where $z$ is the $100(\gamma+1) / 2$-quantile of the standard normal distribution, so that the interval $\left[u_{\mathrm{L}}(t), u_{\mathrm{U}}(t)\right]$ provides probability bands for the mean $y(t)$. For a number of days the retention is a sum of r.v., which means that a good approximation to the normal distribution with a symmetric interval may be used:

$$
P\left[u_{1}(t)-b(t) \leq y(t) \leq u_{1}(t)+b(t)\right]=\gamma,
$$

that is, $b(t) \approx z \sqrt{u_{2}(t)-u_{1}(t)^{2}}$. Some kind of probability region can be built for the stochastic process $y(t)$ :

$$
y(t) \approx u_{1}(t) \pm z \sqrt{u_{2}(t)-u_{1}(t)^{2}} .
$$

We can use these equations to evaluate $y(t)$ and its uncertainties for workers exposed to a daily intake that can be described using a log-normal distribution.

\section{ESTIMATION OF THE INTAKE WITH STATIC AIR SAMPLERS}

The fact that the individual intake could be represented by a log-normal distribution led us to study the method applied in the Juzbado Fuel Fabrication Plant to estimate the intake. This method is similar to that applied in other uranium facilities. The uranium aerosol concentrations are periodically monitored with a Static Air Sampler (SAS). The SAS aspirates the air from the environment using a pump. The air is led through a paper filter where the aerosols are collected. Air samplers are fixed in locations $P_{\mathrm{i}}$ strategically located in the workplace (see Fig. 1).

The filter at point $P_{\mathrm{i}}$ is collected when the workers change shift every working day $j$ and the activity $A_{\mathrm{i}}(j)$ on the filter is measured at the end of the sampling period $T$ (usually an 8-h shift). For special operations where the maximum level of airborne uranium concentration could be exceeded, i.e., while cleaning equipment, individual respirators are used. We will not consider the air concentration during these special periods of time. The daily average concentration $C_{\mathrm{i}}(j)$ for day $j$ at point $P_{\mathrm{i}}$ is given by $C_{\mathrm{i}}(j)=A_{\mathrm{i}}(j) /(\rho T)$, where $\rho$ is the flow through the filter $\left(\rho=1.2 \mathrm{~m}^{3} \mathrm{~h}^{-1}\right.$ here). The worker stays every day, $j$, in $n$ different points, $i$, for a period of time $\Delta t_{\mathrm{ij}}$. The intake for this worker during day $j$ is then $I_{\mathrm{j}} \approx v \sum_{\mathrm{i}=1}^{\mathrm{n}}$ $C_{\mathrm{i}}(j) \Delta t_{\mathrm{ij}}$, where $v$ is the worker's breathing rate. We used " $\approx$ " and not "=" because the concentration $C_{\mathrm{i}}(j)$ is in fact the average concentration at $i$ for the whole day $j$ (not only during the period $\Delta t_{\mathrm{ij}}$ ). Therefore, the intake for a worker during the day $j$ at area $i$ will be

$$
I_{j}=\frac{v}{\rho T} \sum_{i=1}^{n} A_{i}(j) \Delta t_{i j},
$$

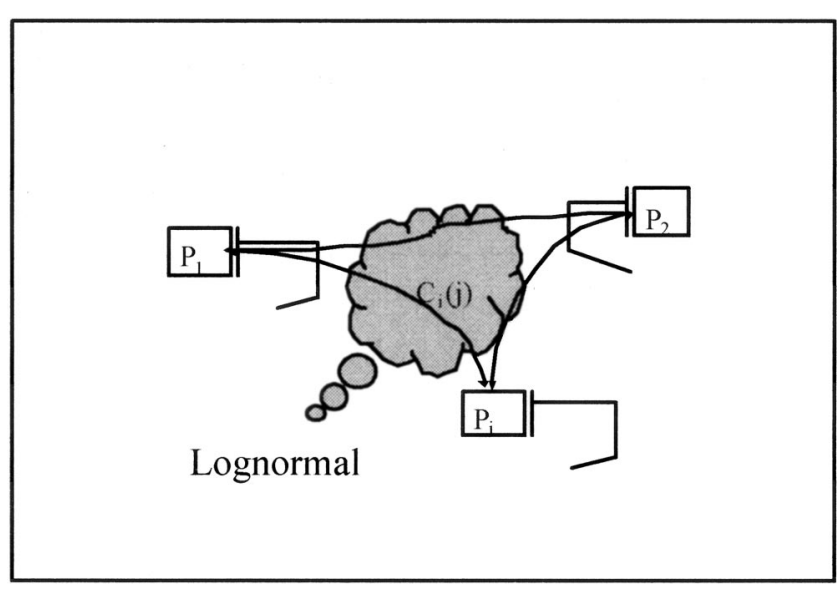

Fig. 1. Worker moving in an area with air samplers fixed at points $P_{\mathrm{i}}$. The daily concentration $C_{\mathrm{i}}(j)$ at point $i$ on day $j$ can be fitted to a log-normal distribution. 
where the worker's breathing rate will be assumed constant $\left(v=1.2 \mathrm{~m}^{3} \mathrm{~h}^{-1}\right)$ for all workers. Denoting $w_{\mathrm{ij}}=$ $\Delta t_{\mathrm{ij}} / T$ the fraction of time the worker stays at point $P_{\mathrm{i}}$ on day $j$ and taking $v=\rho$ then:

$$
I_{j}=\sum_{i=1}^{n} w_{i j} A_{l}(j) .
$$

Eqn (5) gives the intake retention of a worker. After $t$ days of exposure, the retention in one compartment will be

$$
\begin{aligned}
y(t)=\sum_{j=1}^{t} r(t-j+ & 1) \sum_{i=1}^{n} w_{i} A_{i}(j) \\
& =\sum_{i=1}^{n} \sum_{j=1}^{t} A_{i}(j) w_{i} r(t-j+1) .
\end{aligned}
$$

Thus, $w_{\mathrm{i}} r(t-j+1) A_{\mathrm{i}}(j)$ follows a log-normal distribution $L N\left[m_{\mathrm{ij}}(t)+\mu_{\mathrm{i}}, \sigma_{1}^{2}\right]$, where

$$
m_{i j}(t)=\ln \left[w_{i} r(t-j+1)\right] .
$$

Wilkinson's approximation for $y(t)$ provides a lognormal distribution $L N\left[\mu(t), \sigma^{2}(t)\right]$ with first and second moments about the origin

$$
u_{1}(t)=E[y(t)]=\sum_{i, j} \exp \left[m_{i j}(t)+\mu_{i}+\sigma_{i}^{2} / 2\right],
$$

$$
\begin{aligned}
u_{2}(t)= & E\left[y(t)^{2}\right] \\
= & \sum_{i, j} \exp \left\{2\left[m_{i j}(t)+\mu_{i}+\sigma_{i}^{2}\right]\right\} \\
& +\sum_{(i, j) \neq\left(i^{\prime}, j^{\prime}\right)} \sum_{i^{\prime}, j^{\prime}} \exp \left[m_{i j}(t)+m_{i^{\prime}, j^{\prime}}(t)+\mu_{i}+\mu_{i^{\prime}}\right. \\
& \left.+\left(\sigma_{i}^{2}+\sigma_{i^{\prime}}^{2}\right) / 2\right] .
\end{aligned}
$$
obtain:

Then, substituting $m_{\mathrm{ij}}(t)$ in eqn (23) and eqn (24) we

$$
\begin{gathered}
u_{1}(t)=\left(\sum_{i} w_{i} e^{\mu_{i}+\sigma_{i}^{2} / 2}\right) \sum_{j} r(j)=\left(\sum_{i} w_{i} \mu_{l, i}\right) \sum_{j} r(j), \\
u_{2}(t)=\left(\sum_{i} w_{i} e^{\mu_{i}+\sigma_{i}^{2} / 2}\right)^{2}\left(\sum_{j} r(j)\right)^{2} \\
+\sum_{i} w_{i}^{2} e^{2 \mu_{i}+\sigma_{i}^{2}}\left(e^{\sigma_{i}^{2}}-1\right) \sum_{j} r^{2}(j)
\end{gathered}
$$

$$
\begin{aligned}
& =u_{1}(t)^{2}+\sum_{i} w_{i}^{2} e^{2 \mu_{i}+\sigma_{i}^{2}}\left(e^{\sigma_{i}^{2}}-1\right) \sum_{j} r^{2}(j) \\
& =u_{1}(t)^{2}+\sum_{i} w_{i}^{2} \sigma_{I, i}^{2} \sum_{j} r^{2}(j),
\end{aligned}
$$

where $\mu_{\mathrm{I}, \mathrm{i}}$ and $\sigma_{\mathrm{I}, \mathrm{i}}^{2}$ are the mean and variance of the log-normal distribution of the intake in area $i$. We can replace eqn (25) and eqn (26) in eqn (18) to evaluate $y(t)$ and its uncertainties obtaining eqn (27):

$$
\begin{aligned}
y(t) \approx & \left(\sum_{i} w_{i} e^{\mu_{i}+\sigma_{i}^{2} / 2}\right) \sum_{j} r(j) \\
& \pm z \sqrt{\sum_{i} w_{i}^{2} e^{2 \mu_{i}+\sigma_{i}^{2}}\left(e^{\sigma_{i}^{2}}-1\right) \sum_{j} r^{2}(j)} \\
= & \left(\sum_{i} w_{i} \mu_{I, i}\right) \sum_{j} r(j) \pm z \sqrt{\sum_{i} w_{i}^{2} \sigma_{I, i}^{2} \sum_{j} r^{2}(j)},
\end{aligned}
$$

\begin{tabular}{|c|c|c|c|c|c|}
\hline \multirow[b]{2}{*}{ Isotope } & \multirow[b]{2}{*}{$\% \mathrm{wt}^{\mathrm{a}}$} & \multicolumn{2}{|c|}{ Activity $^{\mathrm{b}}$} & \multirow{2}{*}{$\begin{array}{c}\mathrm{DCF}^{\mathrm{c}} \\
\mathrm{mSv} / \mathrm{Bq}\end{array}$} & \multirow{2}{*}{$\begin{array}{r}\operatorname{LLD}^{\mathrm{d}} \\
\mathrm{Bq}\end{array}$} \\
\hline & & $\mathrm{kBq} / \mathrm{g} \mathrm{U}$ & $\%$ & & \\
\hline${ }^{238} \mathrm{U}$ & 95.56 & 11.89 & 10.99 & $5.70 \times 10^{-3}$ & \\
\hline${ }^{235} \mathrm{U}$ & 4.40 & 3.52 & 3.25 & $6.10 \times 10^{-3}$ & 3.0 \\
\hline${ }^{234} \mathrm{U}$ & 0.04 & 92.77 & 85.76 & $6.80 \times 10^{-3}$ & \\
\hline U-total & 100.00 & 108.18 & 100.00 & $6.66 \times 10^{-3}$ & 92.3 \\
\hline
\end{tabular}

where $z$ is the $(1+\gamma) / 2$-quantile of the standard normal distribution.

\section{APPLICATIONS}

In this section the theoretical results given above are applied to bioassay programs. All examples are referred to workers exposed to intakes by inhalation of $\mathrm{UO}_{2}$ (class $\mathrm{S}$ ), with enrichment $4.4 \%$ by weight of ${ }^{235} \mathrm{U}$ and an activity median aerodynamic diameter (AMAD) equal to $5 \mu \mathrm{m}$. Table 1 gives some radioactive characteristics for this type of uranium.

\section{Example 1}

A worker has been exposed during the last 2,000 d to an intake represented by a log-normal distribution with

Table 1. Some radioactive characteristics for uranium enriched $4.4 \%$ in ${ }^{235} \mathrm{U}$.

${ }^{a}$ Experimental values from Fabrica de Juzbado.

${ }^{\mathrm{b}}$ Obtained using (a) and the decay constant values given in ICRP 78 (1997).

${ }^{\mathrm{c}}$ Dose conversion factor for AMAD $=5 \mu \mathrm{m}$ and class S (ICRP 2001).

${ }^{d}$ Typical LLD for the lung counter of the CIEMAT used in routine measurements of Fabrica de Juzbado workers. This value is very close to the best measurements reported in the literature (e.g., Kramer et al. 2003). The LLD for U-total has been obtained assuming that the uranium is enriched $4.4 \%$. 
mean $\mu_{\mathrm{I}, \mathrm{e}}=3.3 \mathrm{~Bq} \mathrm{U}$ and standard deviation $\sigma_{\mathrm{I}, \mathrm{e}}=5.1$ $\mathrm{Bq} \mathrm{U}$, estimated with the individual working day intakes. We wish to know whether the lung burden will exceed the lower limit of detection (LLD), $92 \mathrm{~Bq} \mathrm{U}$ in this case (see Table 1). It is assumed that there is no intake during the weekends, i.e., it will be assumed that $I_{\mathrm{j}}=0$ when $j=7 k$ and $j=7 k-1, k=1,2, \ldots$

Here we use the lung intake retention function for uranium $\left({ }^{238} \mathrm{U},{ }^{235} \mathrm{U},{ }^{234} \mathrm{U}\right)$ type $\mathrm{S}$ and $\mathrm{AMAD}=5 \mu \mathrm{m}$ (Sanchez and Lopez-Fidalgo 2003):

$$
\begin{aligned}
r(t)= & 0.01009 \boldsymbol{e}^{-10.0 t}+0.007959 \boldsymbol{e}^{-2 t} \\
& +0.01031 \boldsymbol{e}^{-0.0301 t}+0.01614 \boldsymbol{e}^{-0.0201 t} \\
& +0.03191 \boldsymbol{e}^{-0.0011 t}+0.004430 \boldsymbol{e}^{-0.00022 t} \\
& +0.001087 \boldsymbol{e}^{-0.0001 t}
\end{aligned}
$$

Eqns (12), (13), and (17) provide the mean and the probability region as follows:

$$
\begin{aligned}
\sum_{j=1}^{2000} r(j)=25.795, & \sum_{j=1}^{2000} r^{2}(j)=0.607, \\
u_{1}(2,000) & =3.3 \sum_{j=1}^{2000} r(j)=85.1 .
\end{aligned}
$$

Then for $\gamma=0.95$ (one-tail interval) and $z_{\gamma}=1.645$ the $\gamma$-quantile of the standard normal distribution

$$
\begin{aligned}
y(2,000) & \approx u_{1}(2,000)+z_{\gamma} \sqrt{u_{2}(2,000)-u_{1}(2,000)^{2}} \\
& =u_{1}(2,000)+z_{\gamma} \sigma_{1} \sqrt{\sum_{j} r^{2}(2,000)} \\
& =85.1+1.645(5.1 \sqrt{0.607})=91.6 \mathrm{~Bq},
\end{aligned}
$$

which is practically the lung counter LLD (see Table 1). For this worker the lung retention and its uncertainties are plotted in Fig. 2. It can be observed that the worker reaches the LLD approximately 2,040 d after starting the intake.

Remark. The average committed effective dose equivalent per year for this worker is $3.3 \mathrm{~Bq}$ U/working day $(365 \mathrm{~d} / \mathrm{y})\left(5\right.$ working d/7 d) $\left(6.66 \times 10^{-3} \mathrm{mSv} / \mathrm{Bq}\right.$ $\mathrm{U})=5.7 \mathrm{mSv} \mathrm{y}^{-1}$. The LLD indicated in ICRP 78 (Table A.10.5) for the lung is $200 \mathrm{~Bq}$ for ${ }^{235} \mathrm{U}$ or approximately $6,200 \mathrm{~Bq} \mathrm{U}$ (for a $4.4 \%$ enrichment), which is 67.4 times $(6,200 \mathrm{~Bq} \mathrm{U} / 92 \mathrm{~Bq} \mathrm{U})$ greater than the LLD used in the example. Consequently, the minimum dose detected after $2,000 \mathrm{~d}$ of the intake would be $\left(5.7 \mathrm{mSv} \mathrm{y}^{-1}\right)(67.4)=$ $384 \mathrm{mSv} \mathrm{y}{ }^{-1}$, which is much greater than the annual worker dose limit of $20 \mathrm{mSv}$, established in ICRP 60 (1991). Therefore a whole body counter with an LLD of $6,200 \mathrm{~Bq} \mathrm{U}$ (corresponding to $200 \mathrm{~Bq}{ }^{235} \mathrm{U}$ for a $4.4 \%$ enrichment) is not applicable for routine analysis.

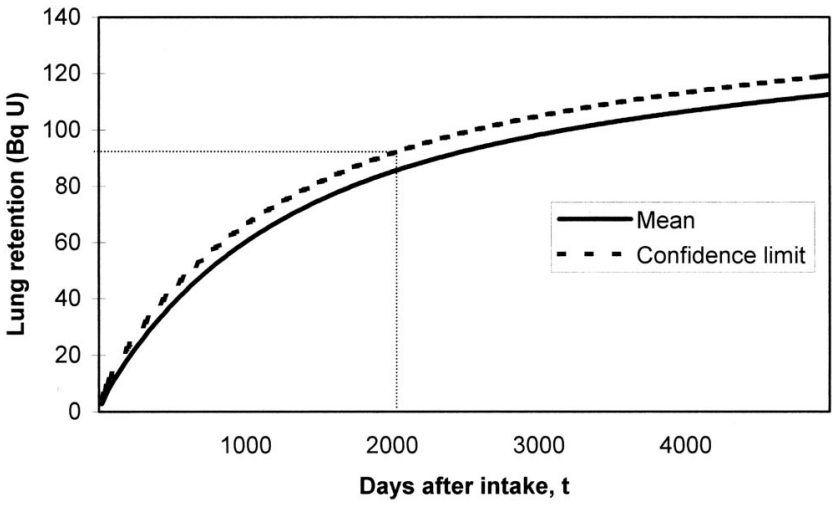

Fig. 2. Predicted values for a log-normal random intake $\left(\mu_{1, \mathrm{e}}=3.3\right.$ $\mathrm{Bq}$ and $\left.\sigma_{\mathrm{I}, \mathrm{e}}=5.1 \mathrm{~Bq}\right)$ and the probability bands with $\gamma=0.95$ for the lung burden of a worker exposed to a random intake. The LLD of $92 \mathrm{~Bq} \mathrm{U}$ for lung counting also is shown in the graph.

Example 2. The intake for a group of workers in the same workshop is estimated by using a SAS placed at three locations. The activity, measured in $\mathrm{mBq}$ at these locations, at the change of shift time can be fitted to $L N(5.56,1.26)$, $L N(5.01,1.5)$, and $L N(3.14,0.86)$, respectively. It was assumed that the proportion of time a worker spends at each location is constant each day: $0.35,0.45$, and 0.20 , respectively. We want to establish the frequency with which a urine sample should be taken. The following criterion established in the Regulatory Guide 8.9 (U.S. NRC 1993) will be applied: "In general, spot samples should be collected frequently enough that there is no more than a $30 \%$ increase in the IRF between bioassay measurements." Here we use the daily urine excretion function for uranium $\left({ }^{238} \mathrm{U}\right.$, ${ }^{235} \mathrm{U},{ }^{234} \mathrm{U}$ ) type $\mathrm{S}$ and $\mathrm{AMAD}=5 \mu \mathrm{m}$ (Sanchez and Lopez-Fidalgo 2003):

$$
\begin{aligned}
r(t)= & -1.5 \mathbf{e}^{-10 t}-1.7 \mathbf{e}^{-6.0 t}+1.1 \mathbf{e}^{-5.4 t} \\
& +0.00016 \mathbf{e}^{-2.0 t}+2.7 \times 10^{-6} \mathbf{e}^{-0.34 t}+3.5 \\
& \times 10^{-6} \mathbf{e}^{-0.14 t}+6.4 \times 10^{-7} \mathbf{e}^{-0.099 t}+1.6 \\
& \times 10^{-5} \mathbf{e}^{-0.097 t}+3.2 \times 10^{-6} \mathbf{e}^{-0.030 t}+2.1 \\
& \times 10^{-6} \mathbf{e}^{-0.020 t}+1.0 \times 10^{-6} \mathbf{e}^{-0.013 t}+3.1 \\
& \times 10^{-6} \mathbf{e}^{-0.0011 t}+4.3 \times 10^{-7} \mathbf{e}^{-0.00022 t}+1.2 \\
& \times 10^{-7} \mathbf{e}^{-0.0001 t} .
\end{aligned}
$$

Here it is worth mentioning that the use of the intake retention function $r(t)$ is applicable not only to lung retention but also to the daily urine and fecal excretion, see, e.g., Skrable et al. (1988), and in this sense $r(t)$ is used in this paper. In fact, the lung and whole body retention as well as the daily urine and fecal excretion after a time $t$ are sums of the contents of several compartments. 
The solution of the equation

$$
\begin{gathered}
\frac{u_{1}(t+T)+z_{(\gamma+1) / 2} \sqrt{u_{2}(t+T)-u_{1}(t+T)^{2}}}{u_{1}(t)} \\
=\frac{u_{1}(t+T)+z_{(\gamma+1) / 2} \sqrt{\sum_{i} w_{i}^{2} \sigma_{I, i}^{2} \sum_{j=1}^{j=1+T} r^{2}(j)}}{u_{1}(t)} \\
=1.30
\end{gathered}
$$

at a specific value of $t$ provides the time $t+T$ for the next measurement. That is, $t$ is the time after the intake when a measurement (a urine sample in this case) is taken. The time from this moment to the time when the next measurement should be made is $T$. The solution of eqn (29) will be applied for each worker. For instance, if a measurement is made $1,100 \mathrm{~d}$ after the intake started, the next one should be made about $T=130 \mathrm{~d}$ later. This situation is illustrated in Fig. 3 considering the time $T$ for which the retention may increase $30 \%$ from the mean value at time $t$ to the upper probability bound.

\section{CONCLUSION}

ICRP Publication 78 (ICRP 1997) requires that the monitoring of the intake of radioactive material and bioassay programs should take into account the intake uncertainties, but it does not establish either precise guidelines on how these uncertainties should be evaluated or how the frequency of the bioassays may be set up. We provide a formula for forecasting the future intake and its uncertainties. This method can be useful to determine when a

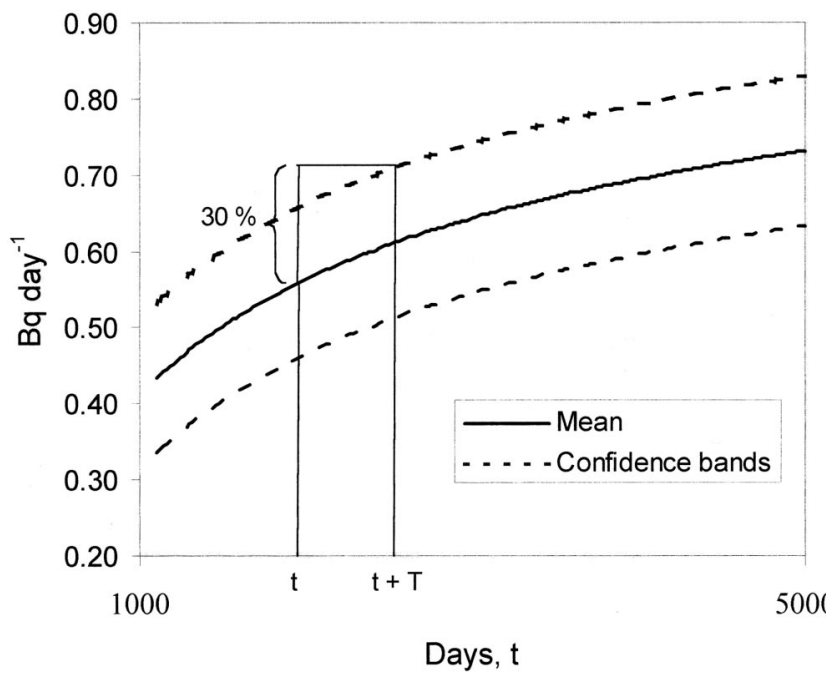

Fig. 3. Probability bands for $\gamma=0.95$ for the urine daily excretion of a worker exposed to a random intake as a function of elapsed time $t$ after the intake. bioassay is required as well as the frequency in its application.

Acknowledgements-This work has been supported by the grant from Junta de Castilla y Leon SA004/01 and by ENUSA Industrias Avanzadas, S.A.

\section{REFERENCES}

Abu-Dayya AA, Beaulieu NC. Outage probabilities in the presence of correlated log-normal interferers. IEEE Trans Veh Technol 43:164-173; 1994.

Cardieri P, Rappaport TS. Statistical analysis of cochannel interference in wireless communications systems. Wirel Commun Mob Comput 1:111-121; 2001.

Fenton LF. The sum of log-normal probability distributions in scatter transmission systems. IRE Trans on Communications Systems 8:57-67; 1960.

Ho C-L. Calculating the mean and variance of power sums with two log-normal components. IEEE Trans Veh Technol 44:756-762; 1995.

International Commission on Radiological Protection. 1990 recommendations of the International Commission on Radiological Protection. Oxford: Elsevier Ltd.; ICRP Publication 60; 1991.

International Commission on Radiological Protection. Individual monitoring for internal exposure of workers. Oxford: Pergamon Press; ICRP Publication 78; 1997.

International Commission on Radiological Protection. ICRP Database of Dose Coefficients: Workers and Members of the Public. Version 2.0.1 (CD-ROM). Oxford: Pergamon Press; ICRP; 2001.

Kercher JR, Robison WL. Uncertainties in predicted radionuclide body burdens doses from discrete stochastic source terms. Health Phys 65:47-68; 1993.

Kramer GH, Hauck BM, Allen SA, Dantas BM, Dantas AL, Azeredo A. Lung counting: summing techniques to reduce the MDA. Health Phys 85:220-227; 2003.

Potter CA. Intake retention fractions developed from models used in the determination of dose coefficients developed for ICRP Publication 68 -Particulate inhalation. Health Phys 83:594-789; 2002.

Sanchez G. BIOKMOD: A Mathematica toolbox for modeling biokinetic systems. Mathematica in Education and Research 10:50-70; 2005.

Sanchez G, Lopez-Fidalgo J. Mathematical techniques for solving analytically large compartmental systems. Health Phys 85:184-193; 2003.

Schwartz S, Yeh YS. On the distribution function and moments of power sums with log-normal components. Bell Syst Tech J 61:1441-1462; 1982 .

Skrable KW, Chabot GE, French CS, La Bone TR. Intake retention functions and their applications to bioassay and the estimation of internal radiation doses. Health Phys 55:933-950; 1988.

U.S. Nuclear Regulatory Commission. Acceptable concepts, models, equations, and assumptions for a bioassay program. NRC: Washington; Regulatory Guide 8.9, Revision 1; 1993. Available at: http://www.nrc.gov/reading-rm/doc-collections/ reg-guides/occupational-health/active. Accessed 14 September 2004. 